JOURNAL DE PHYSIQUE

Colloque C2, supplément au n³, Tome 44, mars 1983

page $\mathrm{C} 2-171$

\title{
INSTABILITIES INDUCED BY RESONANT ABSORPTION OF AN ELECTROMAGNETIC WAVE IN AN INHOMOGENEOUS PLASMA
}

\author{
J.C. Adam, A. Gourdin Servenière and G. Lava1 \\ Centre de Physique Théorique de l'Ecole Polytechnique ${ }^{+}$, Plateau de Palaiseau, \\ 91128 Palaiseau Cedex, France
}

Résumé - Dans le cas d'une incidence oblique, une onde électromagnétique cohérente gënère dans un plasma inhomogène des ondes longitudinales. Ces ondes longitudinales se propagent non-linéairement dans le plasma pour des flux d'intensité modérêe. Ces non-1inêarités sont responsables d'instabilités paramétriques couplant les ondes de Langmuir aux ondes ioniques. On observe alors une transition entre des comportements périodiques et chaotiques correspondant à des émissions de solitons. La conséquence de ce comportement sur les ondes réfléchies et le plasma sont discutés.

\begin{abstract}
For oblique incidence and appropriate polarization, a coherent electromagnetic wave generates longitudinal Langmuir waves in an inhomogeneous plasma. These longitudinal waves propagate non-1inearly through the plasma even for rather low incident energy flux. This non-linearity induces instabilities by coupling with two other Langmuir waves and an ion sound waves. For weak non-linearity or strong damping, periodic time dependent solutions are found. They correspond to periodic emissions of soliton-1ike perturbations. For stronger non-linearity, the solitons are emitted in a chaotic way. The consequence of these behaviors on the reflected electromagnetic wave and the plasma will be discussed.
\end{abstract}

\section{I - INTRODUCTION}

In an inhomogeneous plasma, when the electric field of an electromagnetic wave has a component along the density gradient, large amplitude longitudinal waves are excited at points where the plasma frequency coincides with the wave frequency. This mechanism is called resonant absorption. It takes place for obliquely incident $p$ polarized waves. The large amplitude longitudinal wave can be modulationally unstable. The absorption mechanism becomes non-stationary. We describe here the coupling between these unstable longitudinal waves and the electromagnetic waves.

II - THE ABSORPTION MECHANISM

A plane electromagnetic wave is supposed to be obliquely incident on an inhomogeneous plasma. The plasma frequency $\omega_{p}(x)$ coincides with the wave frequency $\omega_{0}$ for $x=0$ and is an increasing function of $x$ such that $\omega_{p}(x) \rightarrow 0$ as $x \rightarrow-\infty$.

As in Ref. [ 1 ], the wave vector $\vec{k}_{0}$ of the incident wave is lying in the $x, y$ plane and the electric field is contained into the same plane. We assume

$$
\left|\frac{l}{k_{0}} \frac{d}{d x} L_{n} \omega_{p}\right| \ll 1 \quad k_{o} \lambda_{D} \ll 1
$$

where $\lambda_{D}$ is the Debge length.

When collisional and thermal effects are negligible, the cold plasma approximation can be used so that we may write

\footnotetext{
+ "Groupe de Recherche du C.N.R.S. n" 48 "
} 


$$
\vec{J}=i \varepsilon_{0} \frac{\omega_{\mathrm{p}}^{2}}{\omega_{0}} \overrightarrow{\mathrm{E}} \quad \text { with } \quad \overrightarrow{\mathrm{E}}=\overrightarrow{\mathrm{E}}_{0} e^{-i \omega_{0} t}
$$

and the equation for the $\mathrm{B}_{2}$ magnetic field is

$$
\partial_{x} \varepsilon^{-1} \partial_{x} B_{z}+\varepsilon^{-1} \partial^{2}{ }_{y}^{2} B_{z}+k_{o}^{2} B_{z}=0
$$

with $\varepsilon=1-\omega_{\mathrm{p}}^{2} / \omega^{2}$.

By assuming $B_{z}=B(x) e^{-i k_{0} \sin \theta y-i \omega t}$, Equation (2) becomes

$$
\partial_{x} \varepsilon^{-1} \partial_{x} B+k_{0}^{2}\left[1-\varepsilon^{-1} \sin ^{2} \theta\right] B=0
$$

Such an equation is singular for $\mathrm{x}=0$. In the vicinity of this point, Eq. 3 has a regular solution $y_{1}$ and a singular one $y_{2}$. In order to find their behavior in this region, we set $E=-x / L$ in Eq. 3 where $L$ is the local density gradient length. We find

$$
\begin{aligned}
& y_{1}=k_{0}^{2} x^{2}\left[1+\frac{k_{0}^{2} \sin ^{2} \theta}{8} x^{2}+\frac{k_{0}^{2} L^{-1}}{15} x^{3}+\ldots\right] \\
& y_{2}=\sin ^{2} \theta y_{1} \operatorname{Ln}\left(k_{0} x\right)+2\left[1+\frac{k_{0}^{2} L^{-1}}{3} x^{3}+\ldots\right]
\end{aligned}
$$

The appropriate boundary conditions cannot be fulfilled by $y_{1}$ since by multiplying Eq. 3 by $\mathrm{B}^{*}$ and integrating on $\mathrm{x}$ we obtain

$$
\int_{x_{0}}^{x_{1}} d x\left\{\varepsilon^{-1}\left|\frac{\partial B}{\partial x}\right|^{2}-k_{0}^{2}\left[1-\varepsilon^{-1} \sin ^{2} \theta\right]|B|^{2}\right\}=\left[B^{*} \varepsilon^{-1} \frac{\partial B}{\partial x}\right]_{x_{0}}^{x_{1}}
$$

For $x_{1} \rightarrow+\infty$ and $x_{0} \rightarrow 0$, the right hand side vanishes whenever $B=y_{1}$. Thus we have generally $\dot{B}=\lambda y_{1}+\mu y_{2}$ with $\mu \neq 0$. But we also have

$$
E_{x}=-C \sin \theta \varepsilon^{-1} B
$$

and $\mu \neq 0$ implies $B(0) \neq 0$. Thus $E_{x}$ is diverging.

The physical meaning of this divergence results from the resonant excitation of plasma waves at the critical density where $\omega_{0}=\omega_{p}$. The model must be improved in order to broaden this resonance. The excitation of these plasma waves and their subsequent absorption by the plasma is then the dominant absorption mechanism of the incident electromagnetic wave.

\section{III - EQUATIONS FOR THE PLASMA WAVES}

Since the typical scale length of plasma waves is $\lambda_{D}$, conditions (1) insures that we can neglect the $x$ dependence of the electromagnetic field in the plasma wave propagation. For all high frequency quantities $a_{h}$ we set 


$$
a_{h}(x, t)=\operatorname{Re}\left\{a(x, t) \exp \left(-i \omega_{0} t\right)\right\}
$$

where the time dependence of the enveloppe a is slow enough to be neglected in the electromagnetic field equations but at the resonance for the longitudinal waves. Thus we shall be able to take into account the time dependence induced by the instabilities.

We take into account the finite group velocity of longitudinal plasma waves and the low frequency modification of the plasma density $n$ induced by the ponderomotive effect of the high frequency electric field. From

$$
\partial_{y} B_{z}=\mu_{0} j_{x}+\frac{1}{c^{2}} \partial_{t} E_{x}
$$

and

$$
\partial_{t} j_{x}=\left(n_{o}+n_{\ell}\right) \frac{e^{2}}{m_{e}} E_{x}-\frac{3 \varepsilon_{o} T}{m_{e}} \partial^{2}{ }_{x}^{2} E_{x}
$$

we obtain for the fields enveloppes

$$
k_{y} \omega_{o} c^{2} B(0)=\omega_{p e}^{2}\left[\frac{x}{L}+\frac{n_{l}}{n_{0}}\right] E-2 i \gamma \omega_{p} E-3 v_{e}^{2} \partial_{x}^{2} E-2 i \omega_{p} \partial_{t} E
$$

where $E$ is the enveloppe of $E_{x}, \gamma(x)$ a damping rate, $V_{e}$ the electron thermal velocity and where we have used a linear approximation for the $x$ dependence of $\omega^{2}$. The low frequency density perturbation $n_{\ell}$ are given by

$$
\partial_{t}^{2}{ }^{2} n_{\ell}-c_{s}^{2} \partial_{x}^{2}{ }^{2} n_{\ell}=n(0) c_{s}^{2} \partial_{x}^{2} \frac{\varepsilon_{o}|E|^{2}}{n_{c} T}
$$

Knowing $B(0)$, we can solve the non-linear equations ( 10$)$ and (11). However, $B(0)$ is connected to $\mathrm{E}$ as we show in the next section.

IV - CONNECTING FORMULAS

Inside the resonant layer at critical density we have

$$
i \omega_{0} B=\frac{\partial E}{\partial x}-i k_{0} \sin \theta E \text {. }
$$

$B$ is almost constant across the resonant layer and $E$ is very large over a smal1 distance $n$ across $x=0$. Thus we may write.

$$
\left[\mathrm{E}_{\mathrm{y}}\right]_{-\eta}^{+\eta}=-i k_{0} \sin \theta \int_{-\eta}^{+\eta} E(x) d x
$$

For from the resonance, we have again

$$
E_{y}=\frac{c^{2}}{i w_{0} \varepsilon} \partial_{x}^{B}
$$

and with $\varepsilon \simeq-x / L$, we obtain 


$$
\left[\begin{array}{lll}
\frac{1}{x} & \partial_{x} & B
\end{array}\right]_{-\eta}^{+n}=-\frac{k_{0}^{2} \sin \theta}{L C} \int_{-\eta}^{+\eta} E(x) d x
$$

For $x>0$, a solution of Eq. 3 such that $B \rightarrow 0$ for $x \rightarrow \infty$ is of the form

$$
B=\ell y_{1}+y_{2}
$$

where $\ell$ is real.

For $x<0$, a solution of Eq. 3 such that it corresponds for $x \rightarrow-\infty$ to a pure in going wave of amplitude unity and given phase is

$$
B=\alpha y_{1}+B y_{2}
$$

and for a pure outgoing wave,

$$
\mathrm{B}=\alpha^{*} \mathrm{y}_{1}+\beta^{*} \mathrm{y}_{2} .
$$

Using Eq. 5, we find that

$$
\operatorname{Im}\left[\begin{array}{llll}
4 \mathrm{~L} & \beta^{*} & \alpha & \mathrm{k}_{\mathrm{o}}
\end{array}\right]=-1
$$

which is the condition for conservation of the energy flux. If $B_{o}$ and $B_{o}^{\prime}$ are the magnetic component of the incident and reflected wave, the appropriate solution of Eq. 3 for $x<0$ is

$$
B_{-}=\left(B_{o}^{\alpha}+B_{o}^{\prime} \alpha^{*}\right) y_{1}+\left(B_{o}^{\beta}+B_{o}^{\prime} \beta^{*}\right) y_{2}
$$

and for $\mathrm{x}>0$

$$
\mathrm{B}_{+}=p\left(\ell \mathrm{y}_{1}+\mathrm{y}_{2}\right)
$$

We have $B_{-}(0)=B_{+}(0)$ which gives with Eq. 12 :

$$
\begin{gathered}
B_{0}^{\prime}=-B_{0} \frac{\beta \ell-\alpha}{\beta^{*} \ell-\alpha}-\frac{\sin ^{2} \theta}{2 L c\left[\beta^{*} \ell-\alpha^{*}\right]} \int_{-n}^{+n} E d x \\
\rho=+\frac{B(0)}{2}=\frac{\left(\beta^{*} \ell-\alpha^{*}\right)^{-1}}{2 L}\left[\frac{B_{0}}{2 k_{0}}-\frac{\sin ^{2} \theta \beta^{*}}{c} \int E d x\right]
\end{gathered}
$$

For a given $B_{o}$, Eq. 15 provides the value of $B(0)$ to be inserted in Eq. 10 . Solving Eq. 10 determines $E(x)$ from which we obtain the reflected field $B_{0}^{\prime}$ by using Eq. 14 .

$V$ - THE WEAK ABSORPTION CASE

It is well known ${ }^{[2]}$ that the absorption coefficient remains much small than unity unless $\left(k_{0} L\right)^{1 / 3} \sin \theta \sim 1$. In such cases $\rho$ depends only weakly on $E$. Thus we may assume that $B(0)$ is given, does not depend on time and we solve Eqs. 10, 11. This 
approximation has been used 1,3 to obtain stationary solutions. It has been shown that non-1inear profile modifications by ponderomotive effects lead to a reduction of the absorption coefficient a . We find

$$
\frac{a}{a_{0}}=0.8 p^{-0.90} \text { for } 2<p<100
$$

where $a_{0}$ is the linear absorption and

$$
p=\left(\frac{L}{\lambda_{D}}\right)^{2} \frac{\varepsilon_{o}|E d|^{2}}{n_{o} T}
$$

with $E_{d}=\sin \theta \quad C \quad B(0)$.

If Eq. 10 is solved numerically, keeping the time dependence of $E$, it is found that this statior zry solution is unstable for $\mathrm{p}>1$ for all physically relevant values of the parameters. It can be stabilized only by introducing large values of $\gamma$ such that the non-linear effects become negligible and we recover $a \simeq a_{0}$.

For $p \gtrsim 1$, the time dependent solution looks like periodic emission of solitons from the resonant region where $x \simeq 0$ (Fig. 1). These solitons convect towards the low density plasma where they are absorbed by Landau damping. For high values of $p$, the soliton emission becomes chaotic (Fig. 2)

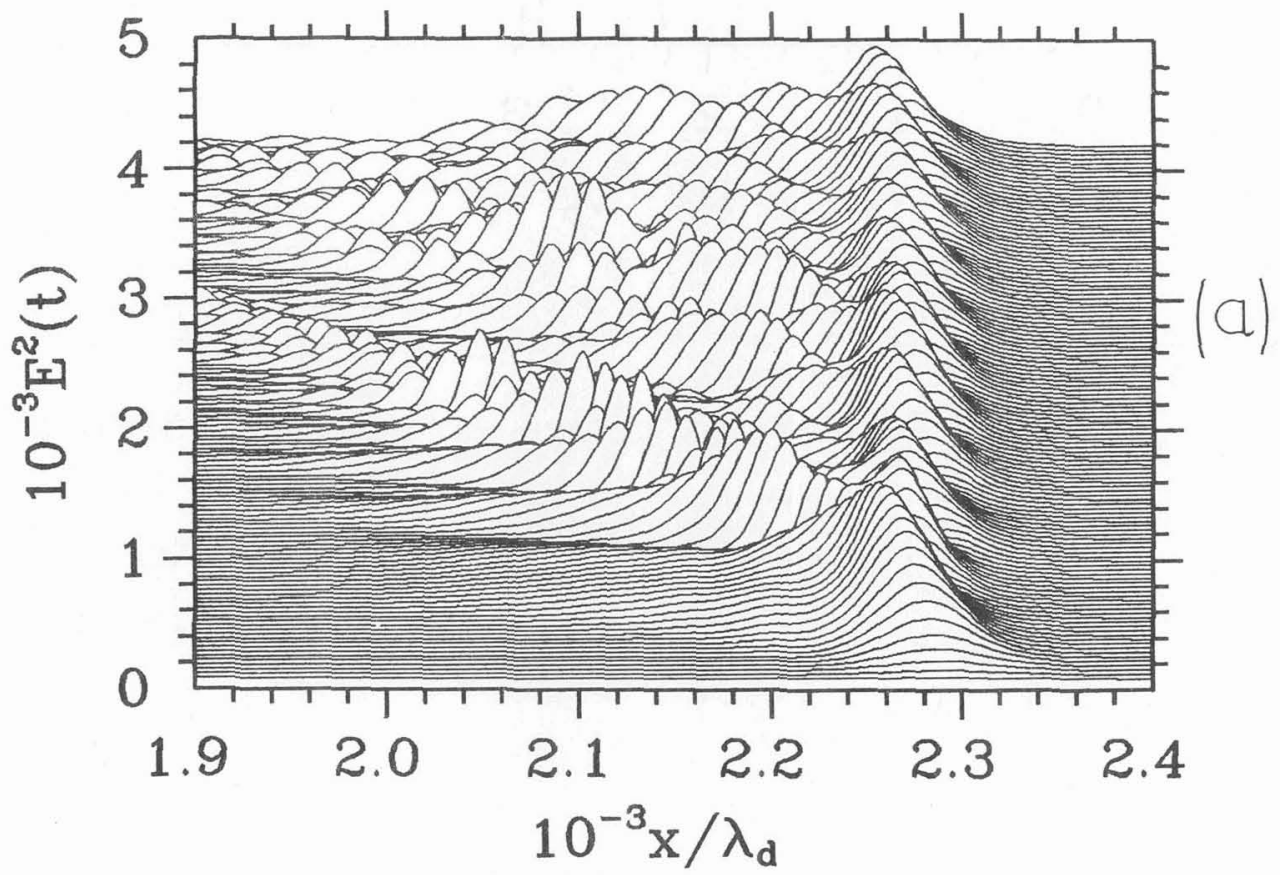




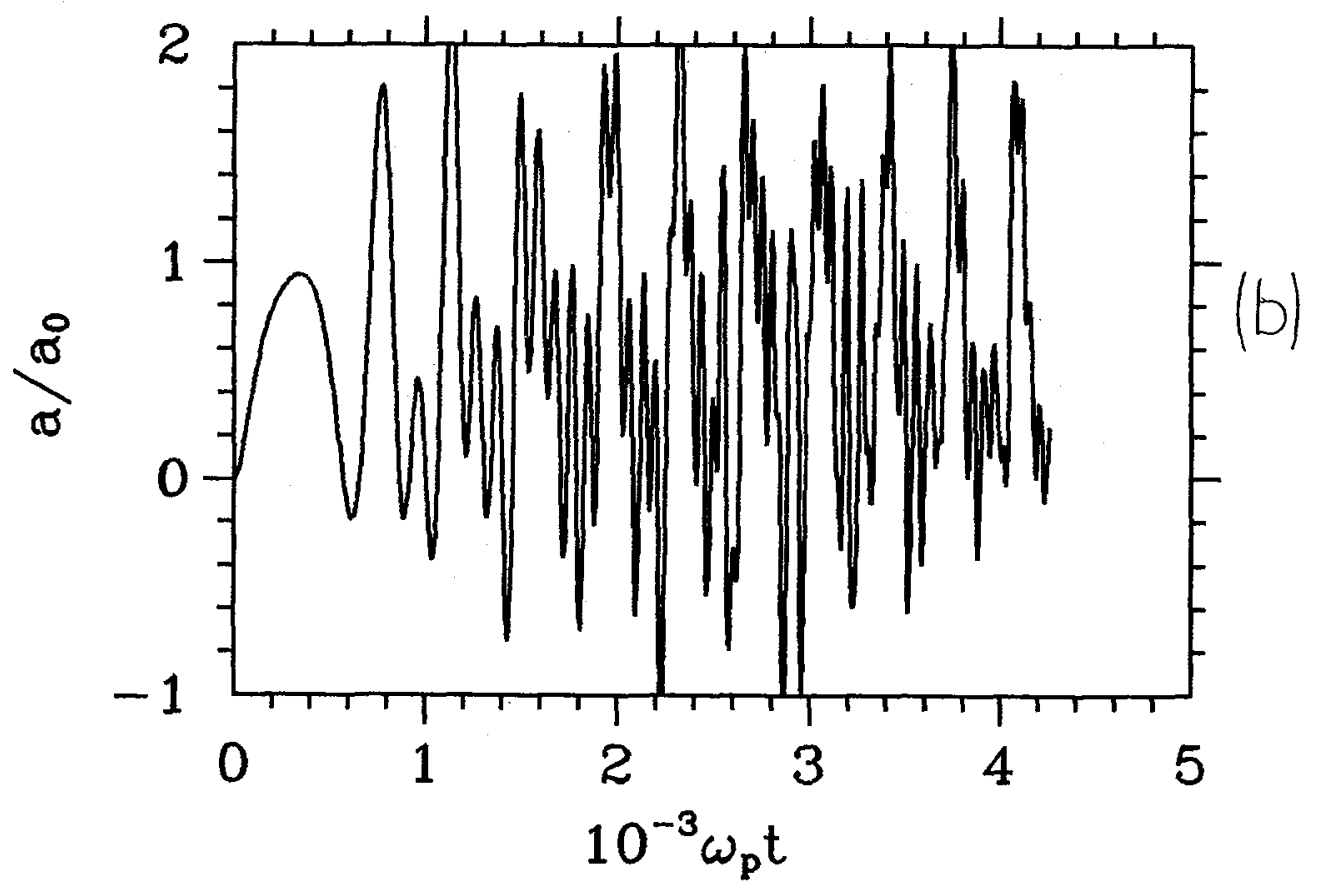

Figures 1 (a) - Space time representation of $E(t)$ for $p=1.33$. Each curve shows the $x$-dependence of $E$ at given time $t$. This time can be found at the intersection of the curve with a vertical axis.

(b) - Spatial average value of $E$ for $p=1.33$ as a function of time. 

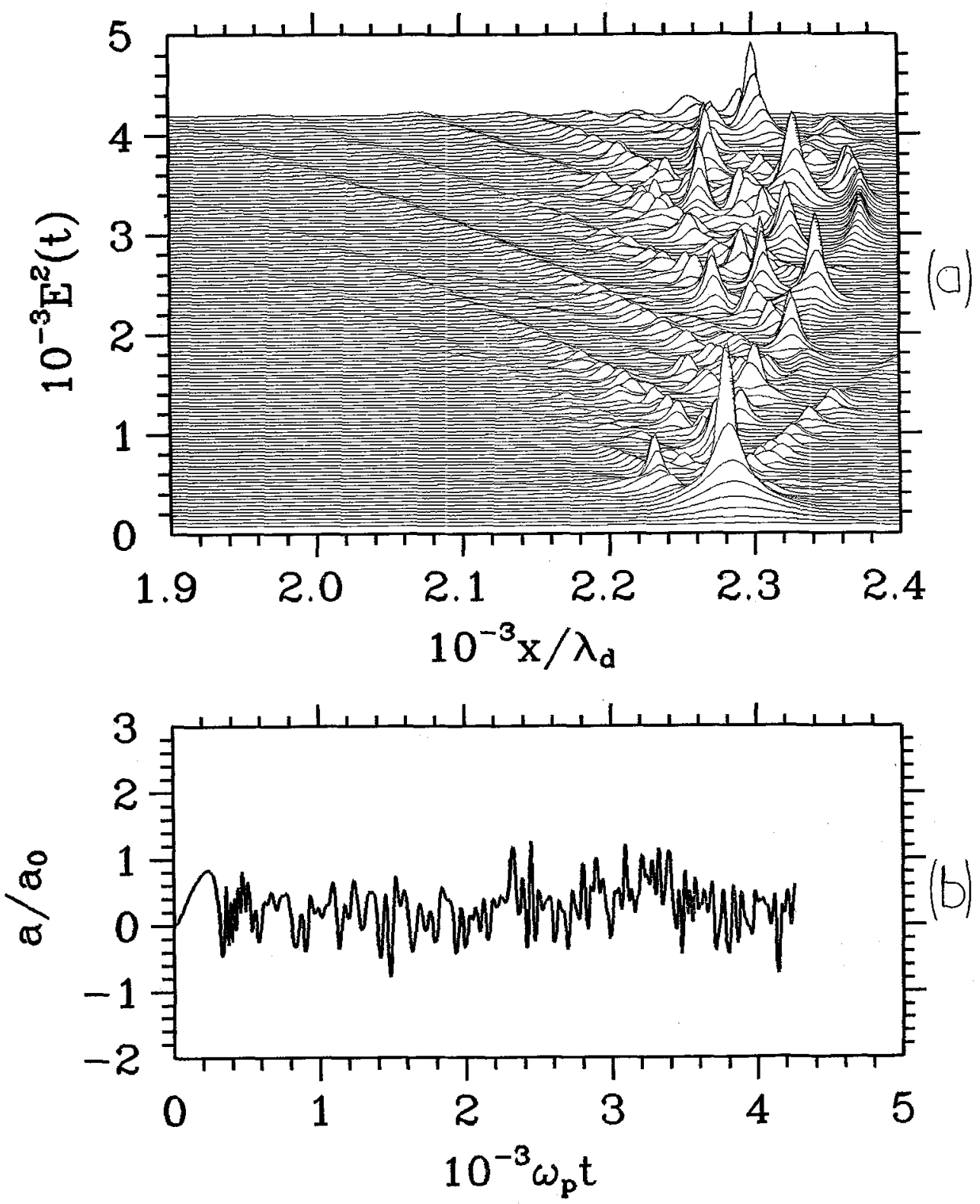

Figures 2 (a) - Space time representation of $E(t)$ for $p=38$. Each curves shows the $x$-dependence of $E$ at given time $t$. This time can be found at the intersection of the curve with a vertical axis.

(b) - Spatial average value of $E$ for $p=38$ as a function of time. 
VI - COUPLING

In order to couple the solutions of Eqs. 10, 11 to the radiation field, we may use Eq. 14 to determine the amplitude of the reflected field. It is easier to determine the absorption coefficient by noticing that it corresponds to the work of the electric field on the plasma particles. We find that the absorbed energy flux is given by.

$$
\pi=\frac{k y}{2 \mu_{0}} \operatorname{Im} \int \mathrm{dx} \mathrm{E}^{*} \mathrm{~B}_{\mathrm{z}}(0)
$$

On Figs. 1 and 2, we have plotted the ratio $a / a_{0}=\pi / \pi_{0}$ where $a$ is the absorption coefficient, $a_{0}$ and $\pi_{0}$ being the values of $a$ and $\pi$ in the usual linear case. It is seen that a oscillates with a frequency much larger than the amplitude time scale. It must be noticed also that for large $p$, the average value of $a$ is larger than the stationary state estimation given by Eq. 16 .

As regards the high frequency oscillation of $a$, we can understand its origin in a particular case. For weak amplitudes of the incident waves and large $I$, we can have

$$
\partial^{2}{ }^{2} \mathrm{n}_{\ell} \ll \mathrm{C}_{\mathrm{s}}^{2}{ }_{\mathrm{x}}^{2} \mathrm{n}_{\ell}
$$

so that Eq. 11 reduces to

$$
n_{\ell}=-n_{o} \partial_{x^{2}}\left(\frac{\varepsilon_{0}|E|^{2}}{n_{c} T}\right)
$$

and Eq. 10 becomes

$$
E_{d}=\left[\frac{x}{L}-\frac{\varepsilon_{o}|E|^{2}}{n_{c} T}\right] E-3 \lambda_{D}^{2} \partial_{x}^{2}{ }^{2} E-\frac{2 i}{\omega_{p}}\left[\partial_{t} E+\gamma E\right]
$$

For $E_{d}=\gamma=0$, Eq. 10 can be transformed into a non-linear Schrödinger equation (4) which can be solved by the inverse scattering method. Numerically, we find that solitons are emitted in the vicinity of the origin. These solitons reach a large amplitude before convecting towards $x \rightarrow-\infty$. Thus a solution of Eq. 19 with $E_{d}=0$ has some relevance with the real problem, since, part of the time, $E_{d}$ and $\gamma^{-}$are sma11 perturbations. Such a solution can be written

$$
E=a e^{-i\left[\delta \omega t+\alpha\left(x t-\lambda^{2} t^{3}\right)\right]} \operatorname{sech}\left[b\left(x+\gamma t^{2}\right)\right]
$$

with $\frac{\delta \omega}{\omega}=-\frac{\varepsilon a^{2}}{2 n_{c} T}, \alpha=\frac{\omega p}{2 L}, \lambda^{2}=\frac{v_{\text {the }}^{2}}{L}, b=\lambda_{D}^{-1} \vee \frac{\varepsilon_{o} a^{2}}{n_{c} T}, \gamma=\frac{\lambda_{D}^{2}}{L} \omega_{p}^{2}$

which describes a soliton centered in $x=0$ at $t=0$ and uniformly accelerated towards the low density. For such a solution we find

$$
\int_{-\infty}^{+\infty} E d x=i e^{-i \delta \omega t} \frac{a}{b} \pi[\operatorname{sh}(\pi \alpha t / b)]^{-1}
$$

Thus, by inserting this result in Eq. 14 we find that such a soliton radiates at a frequency $\omega_{0}+\delta \omega$. The intensity of the radiation do not depend on the soliton amplitude but the duration of the emission is $\left(\varepsilon_{0} a^{2} / n_{c} T\right)^{1 / 2}\left(2 \mathrm{~L} / \lambda_{\mathrm{D}}\right)$. These results 
explain the fast oscillation of a and shows that the reflected light should have a red-shifted component.

\section{CONCLUSION}

When the ponderomotive effect is the dominant non-1inear mechanism, resonant absorption of electromagnetic waves in an inhomogeneous medium is a non stationary process for $p>1$ and weak damping rates. The non-linear evolution shows a transition from a periodic to a disordered regime. Soliton like perturbation plays a dominant role in the absorption mechanism as well as in the frequency spectrum of reflected light.

\section{REFERENCES}

1. ADAM, J.C., GOURDIN-SERVENIERE, A., LAVAL, G., Phys. Fluids 25 (1982) 376.

2. GINZBURG, V.L., The Propagation of Electromagnetic Waves in Plasmas (Pergamon, N.Y. 1970), 2nd Ed.

3. GILDENBURG, V.B., FRAINAN, G.M., Zh. Eksp. Teor. Fiz. 69 (1975) 1601

Sov. Phys. J.E.T.P. $4 \overline{2}(1975) 816$.

4. CHEN, H.C., LIU, C.S., Phys. Rev. Lett. 37 (1976) 693. 